\title{
BMO-Teichmüller spaces revisited
}

\author{
Huaying Wei *, Michel Zinsmeister ${ }^{\dagger}$
}

\begin{abstract}
In [6] the equivalence among three definitions of BMOTeichmüller spaces associated with a Fuchsian group was proven using the Douady-Earle extension operator. In this paper, we show that these equivalences are actually biholomorphisms. In [6] it was further shown that the Douady-Earle extension operator is continuous at the origin. We improve this result by showing Gâteaux-differentiability at this point.
\end{abstract}

Key words and phrases strongly quasisymmetric homeomorphisms, Carleson measures, Douady-Earle extension.

2010 Mathematics Subject Classification 30C62, 30F60, 30H35.

\section{Introduction}

Let $h$ be a quasisymmetric homeomorphism of the unit circle $\mathbb{S}$. Ahlfors and Beurling [5] have been the first to prove that $h$ may be extended to a quasiconformal homeomorphism of the unit disk $\mathbb{D}$. Later Douady and Earle [7] found a conformally natural way to extend $h$ to a quasiconformal homeomorphism of $\mathbb{D}$. More precisely, their extension, called Douady-Earle extension (or barycentric extension), denoted by $E(h)$, satisfies

$$
E(\tau \circ h \circ \alpha)=\tau \circ E(h) \circ \alpha
$$

for any couple $\tau, \alpha$ of automorphisms of $\mathbb{D}$. The Douady-Earle extension plays an important role applied to quasisymmetric homeomorphisms of $\mathbb{S}$ in the complex analytic theory of Teichmüller spaces. In this paper, our study on BMO-Teichmüller theory is based on good properties of the Douady-Earle extension.

\footnotetext{
*Department of Mathematics and Statistics, Jiangsu Normal University, Xuzhou 221116, PR China. Email: 6020140058@jsnu.edu.cn. Research supported by the National Natural Science Foundation of China (Grant No. 11501259) and the Natural Science Foundation of Colleges of Jiangsu Province (Grant No. 15KJB110006).

${ }^{\dagger}$ Corresponding author. MAPMO, Université d' Orléans, Orléans Cedex 2, France. Email: zins@univ-orleans.fr
} 
The universal Teichmüller space $T$ can be defined as the space $Q S_{*}(\mathbb{S})$ of all normalized quasisymmetric homeomorphisms of $\mathbb{S}$. In this setting, the Teichmüller projection $\Phi$ is regarded as the boundary extension map on the space $Q C_{*}(\mathbb{D})$ of all normalized quasiconformal homeomorphisms of $\mathbb{D}$. By the measurable Riemann mapping theorem, we can identity the latter space with the space of Beltrami coefficients $M(\mathbb{D})=L^{\infty}(\mathbb{D})_{1}$, which is the open unit ball of measurable functions on $\mathbb{D}$ with the supremum norm. Then $\Phi: M(\mathbb{D}) \rightarrow T$ is continuous with respect to the topology on $Q S_{*}(\mathbb{S})$ induced by the quasisymmetry constant. The Douady-Earle extension yields a continuous section $e: T \rightarrow M(\mathbb{D})$ for $\Phi$. This section is called the Douady-Earle extension operator which maps the quasisymmetric homeomorphism $h$ to the complex dilatation $\mu$ of the Douady-Earle extension $E(h)$ of $h$. The continuity of this section $e$ combined with conformally natural property of the Douady-Earle extension allowed Douady and Earle [7] to give a much simpler proof of the theorem of Tukia [19, 20] stating that the Teichmüller space of any Fuchsian group is contractible.

The universal BMO-Teichmüller space $T_{b}$ is similarly defined as a subspace of $T$. It is defined as the subspace $S Q S_{*}(\mathbb{S}) \subset Q S_{*}(\mathbb{S})$ of all normalized strongly quasisymmetric homeomorphisms. The topology on $S Q S_{*}(\mathbb{S})$ is induced by the BMO norm. On the other hand, the corresponding subspace of Beltrami coefficients is $\mathcal{M}(\mathbb{D}) \subset M(\mathbb{D})$, which consists of all $\mu \in M(\mathbb{D})$ such that

$$
\frac{|\mu|^{2}(z)}{1-|z|^{2}} d x d y
$$

is a Carleson measure in $\mathbb{D}$. Let's consider a Fuchsian group $G$ : define $\mathcal{M}(G)=$ $M(G) \cap \mathcal{M}(\mathbb{D}), S Q S_{*}(G)=Q S(G) \cap S Q S_{*}(\mathbb{S})$. The same equivalence relation as in the classical case may be defined on $\mathcal{M}(G)$ and we denote by $\mathcal{T}_{S}$ the quotient space ( $S$ is the Riemann surface $\mathbb{D} / G$ ). Let $T(G)$ be the space of Schwarzian derivatives of injective holomorphic functions in $\hat{\mathbb{C}} \backslash \overline{\mathbb{D}}$ having a quasiconformal extension to $\mathbb{C}$. Define $\mathcal{T}(G)=\left\{\varphi \in T(G) ;|\varphi|^{2}(z)\left(|z|^{2}-1\right)^{3} d x d y\right.$ is a Carleson measure on $\left.\hat{\mathbb{C}} \backslash \overline{\mathbb{D}}\right\}$.

Cui and Zinsmeister have proved in [6] that for any $h \in S Q S_{*}(\mathbb{S})$ the complex dilatation $\mu$ of its Douady-Earle extension is in $\mathcal{M}(\mathbb{D})$. Based on this well-defined property of the Douady-Earle extension operator restricted in $S Q S_{*}(\mathbb{S})$, Cui and Zinsmeister have shown that the map $\Psi: \mathcal{T}_{S} \rightarrow S Q S_{*}(G)$ is a bijection while the Bers embedding $\beta: \mathcal{T}_{S} \rightarrow \mathcal{T}(G)$ is bijective. In section 3, we will show that complex Banach manifold structures can be provided for $\mathcal{T}_{S}$ and $S Q S_{*}(G)$ through the Bers embedding $\beta$ and the map $\beta \circ \Psi^{-1}$. Then both the map $\Psi: \mathcal{T}_{S} \rightarrow S Q S_{*}(G)$ and the Bers embedding $\beta: \mathcal{T}_{S} \rightarrow \mathcal{T}(G)$ become biholomorphic.

By Cui and Zinsmeister $e(h) \in \mathcal{M}(\mathbb{D})$ if $h \in S Q S_{*}(\mathbb{S})$, and moreover $e$ is continuous at the origin. The global continuity is not known. This property would imply contractibility of all BMO-Teichmüller spaces; So far only the case $G=\{I\}$ is known [8]. In section 4 , it is proven that at least the operator $e$ is also Gâteaux-differentiable at the origin, and we identify its differential which happens to be a very simple operator. 
In section 2, we will explain the above mentioned concepts and results in more detail.

\section{Preliminaries}

In this section, we summarize several results on the background of our arguments. This includes definitions and properties of Teichmüller spaces, preliminaries on BMOTeichmüller spaces and fundamental results on groups of divergence type and groups of convergence type.

2.1 Teichmüller theory. Let $G$ be a Fuchsian group, i.e. a properly discontinuous fixed point free group of Möbius transformations which keeps $\mathbb{D}$ invariant. For such a group we define $M(G)$ as

$$
M(G)=\left\{\mu \in L^{\infty}(\mathbb{D}):\|\mu\|_{\infty}<1 \text { and } \forall g \in G, \mu=\mu \circ g \frac{\overline{g^{\prime}}}{g^{\prime}}\right\} .
$$

For any $\mu \in M(G)$, there exists a unique quasiconformal self-mapping $f^{\mu}$ of $\mathbb{D}$ keeping $1, i$ and -1 fixed and satisfying

$$
\frac{\partial f^{\mu}}{\partial \bar{z}}=\mu \frac{\partial f^{\mu}}{\partial z}
$$

in $\mathbb{D}$. Similarly, there exists a unique quasiconformal homeomorphism of $\hat{\mathbb{C}}$ which is holomorphic in $\mathbb{D}^{*}$ with the normalization

$$
f_{\mu}(z)=z+\frac{b_{1}}{z}+\cdots
$$

at $\infty$ and such that

$$
\frac{\partial f_{\mu}}{\partial \bar{z}}=\mu \frac{\partial f_{\mu}}{\partial z}
$$

in $\mathbb{D}$. If $g$ is a choice of a Riemann mapping from $\mathbb{D}$ onto $\Omega, \Omega=f_{\mu}(\mathbb{D})$, then $f^{\mu}=$ $g^{-1} \circ f_{\mu}$ is the conformal welding with respect to the boundary of the domain $\Omega$. The mappings $f^{\mu}$ and $f_{\mu}$ respectively induce an isomorphism of the group G onto the Fuchsian group

$$
G^{\mu}=\left\{f^{\mu} \circ g \circ\left(f^{\mu}\right)^{-1} \mid g \in G\right\}
$$

and the quasi-Fuchsian group

$$
G_{\mu}=\left\{f_{\mu} \circ g \circ\left(f_{\mu}\right)^{-1} \mid g \in G\right\}
$$

i.e., a Möbius transformation group acting properly discontinuous on the quasidisk $f_{\mu}(\mathbb{D})$.

The mapping $f^{\mu}$ has a geometric interpretation: If we denote by $S$ the Riemann surface $\mathbb{D} / G$, then $f^{\mu}$ is the lift (to the universal covering) of a quasiconformal mapping from the Riemann surface $S$ onto $S^{\prime}=\mathbb{D} / G^{\mu}$. Conversely, if $\mathrm{F}$ is a quasiconformal 
homeomorphism from $\mathrm{S}$ to a Riemann surface $S^{\prime}$, it has a lift to a quasiconformal homeomorphism $f$ of $\mathbb{D}$ and, replacing if necessary $F$ by $\theta \circ F$, where $\theta: S^{\prime} \rightarrow S^{\prime \prime}$ is a conformal isomorphism, we may assume that $f=f^{\mu}$ for some $\mu \in M(G)$.

If $\mu \in M(G)$, then $f^{\mu}$ has a well-defined boundary value which is a quasisymmetric homeomorphism of $\mathbb{S}$. We define an equivalence relation on $M(G)$ by $\mu \sim \nu$ if $\left.f^{\mu}\right|_{\mathbb{S}}=$ $\left.f^{\nu}\right|_{\mathbb{S}}$. Again this equivalence relation has a geometric interpretation: If $\mathrm{F}, \mathrm{G}$ represent the quasiconformal mappings on $\mathrm{S}$ whose lifts are precisely $f^{\mu}$, $f^{\nu}$, then $\mu \sim \nu$ is equivalent to saying that $G \circ F^{-1}$ is homotopic to a conformal isomorphism between $F(S)$ and $G(S)$, the homotopy being constant on the (possibly empty) boundary of $F(S)$.

The Teichmüller space $T_{S}$ is the quotient space $M(G) / \sim$. We refer to [14] for details about this construction.

If $\mu \in M(G)$, then the Teichmüller space $T_{S}$ can be characterized as the set of quasisymmetric homeomorphisms $\left.f^{\mu}\right|_{\mathbb{S}}$. Since $\mu \in M(G)$, the mappings $f^{\mu}$ and $f^{\mu} \circ g$ have the same complex dilatation. It follows that $f^{\mu} \circ g \circ\left(f^{\mu}\right)^{-1}$ is a Möbius transformation. It is well known that $\Psi:\left.[\mu] \mapsto f^{\mu}\right|_{\mathbb{S}}$ is a bijection from $T_{S}$ onto $Q S_{*}(G)$, the set of quasisymmetric homeomorphisms $h$ of $\mathbb{S}$ keeping $1, i$ and -1 fixed and such that $h \circ g \circ h^{-1}$ is a Möbius transformation.

There is a similar description of the Teichmüller space in terms of $f_{\mu}$. Let the Banach space $\mathrm{B}(\mathrm{G})$ be the space consisting of all functions $\varphi$ holomorphic in $\mathbb{D}^{*}$ which are quadratic differentials for $\mathrm{G}$ and have a finite hyperbolic supremum norm:

$$
\|\varphi\|_{B}=\sup _{z \in \mathbb{D}^{*}}|\varphi(z)| \rho_{\mathbb{D}^{*}}^{-2}(z)<\infty
$$

If $\mu \in M(G)$, then the quasiconformal mapping $f_{\mu} \circ g \circ f_{\mu}^{-1}, g \in G$ of the plane is a Möbius transformation. It follows that

$$
\mathcal{S}_{\left.f_{\mu}\right|_{\mathbb{D}^{*}}}=\mathcal{S}_{\left.\left(f_{\mu} \circ g \circ f_{\mu}^{-1}\right) \circ f_{\mu}\right|_{\Delta^{*}}}=\mathcal{S}_{\left.f_{\mu} \circ g\right|_{\mathbb{D}^{*}}}=\left(\mathcal{S}_{\left.f_{\mu}\right|_{\Delta^{*}}} \circ g\right)\left(g^{\prime}\right)^{2}
$$

So the Schwarzian derivative $\mathcal{S}_{\left.f_{\mu}\right|_{\mathbb{D}^{*}}}$ is a quadratic differential for G. It is also well known that the Bers embedding $\beta:[\mu] \mapsto \mathcal{S}_{\left.f_{\mu}\right|_{\mathbb{D}^{*}}}$ is a bijection from $T_{S}$ onto $T(G)$, the space of Schwarzian derivatives of injective holomorphic functions in $\mathbb{D}^{*}$ having a quasiconformal extension to the complex plane which are quadratic differentials for $\mathrm{G}$. It is known that the set $T(G)$ is an open subset in the complex Banach space $B(G)$, and the ball

$$
B(0,2)=\left\{\phi \in B(G):\|\phi\|_{B}<2\right\}
$$

lies in $T(G)$. With the aid of the Bers embedding, $T_{S}$ carries a natural complex structure. The Teichmüller space $T_{S}$ thus becomes a complex analytic Banach manifold. We refer to [14] for details about the Teichmüller theory.

2.2 BMO-Teichmüller theory. Recall that a positive measure $\lambda$ defined in a simply 
connected domain $\Omega$ is called a Carleson measure (see [11]) if

$$
\|\lambda\|_{c}=\sup \left\{\frac{\lambda(\Omega \cap D(z, r))}{r}: z \in \partial \Omega, 0<r<\operatorname{diameter}(\Omega)\right\}<\infty
$$

where $D(z, r)$ is the disk with center $z$ and radius $r$. A Carleson measure $\lambda$ is called a vanishing Carleson measure if $\lim _{r \rightarrow 0} \lambda(\Omega \cap D(z, r)) / r=0$ uniformly for $z \in \partial \Omega$. We denote by $C M(\Omega)$ and $C M_{0}(\Omega)$ the set of all Carleson measures and vanishing Carleson measures on $\Omega$, respectively.

We denote by $\mathcal{L}(\mathbb{D})$ the Banach space of essentially bounded measurable functions $\mu$ on $\mathbb{D}$ such that the measure

$$
\lambda_{\mu}=\frac{|\mu|^{2}(z)}{1-|z|^{2}} d x d y
$$

in $C M(\mathbb{D})$. The norm on $\mathcal{L}(\Delta)$ is defined as

$$
\|\mu\|_{c}=\|\mu\|_{\infty}+\left\|\lambda_{\mu}\right\|_{c}^{1 / 2}
$$

where $\left\|\lambda_{\mu}\right\|_{c}$ is the Carleson norm of $\lambda_{\mu}$ defined in (1). Set $\mathcal{M}(\mathbb{D})=\{\mu \in \mathcal{L}(\mathbb{D})$ : $\left.\|\mu\|_{\infty}<1\right\}$. Define $\mathcal{M}(G)=M(G) \cap \mathcal{M}(\mathbb{D})$. The same equivalence relation as in the classical case may be defined on $\mathcal{M}(G)$ and we denote by $\mathcal{T}_{S}$ the quotient space which can be called BMO-Teichmüller spaces.

An homeomorphism $h$ of $\mathbb{S}$ is called strongly quasisymmetric (see [13]) if there exist two positive constants $C_{1}(h), C_{2}(h)$, called the strongly quasisymmetric constants of $h$ such that

$$
\frac{|h(E)|}{|h(I)|} \leqslant C_{1}(h)\left(\frac{|E|}{|I|}\right)^{C_{2}(h)}
$$

whenever $I \subset \mathbb{S}$ is an interval and $E \subset I$ a measurable subset. In other words, $h$ is strongly quasisymmetric if and only if $h$ is absolutely continuous so that $\left|h^{\prime}\right|$ belongs to the class of weights $A^{\infty}$ introduced by Muckenhoupt, in particular, $\log h^{\prime}$ belongs to $B M O(\mathbb{S})$, the space of integrable functions on $\mathbb{S}$ of bounded mean oscillation. Let $S Q S(\mathbb{S})$ denote the set of all strongly quasisymmetric homeomorphisms on $\mathbb{S}$. We define $S Q S_{*}(G)=Q S_{*}(G) \cap S Q S(\mathbb{S})$.

We denote by $\mathcal{B}\left(\mathbb{D}^{*}\right)$ the Banach space of function $\varphi$ holomorphic in $\mathbb{D}^{*}$ such that the measure

$$
\lambda_{\varphi}=|\varphi(z)|^{2}\left(|z|^{2}-1\right)^{3} d x d y
$$

in $C M\left(\mathbb{D}^{*}\right)$. The norm on $\mathcal{B}\left(\mathbb{D}^{*}\right)$ is

$$
\|\varphi\|_{\mathcal{B}}=\left\|\lambda_{\varphi}\right\|_{c}
$$

Define $\mathcal{T}(G)=\left\{\varphi \in T(G): \lambda_{\varphi} \in C M\left(\mathbb{D}^{*}\right)\right\}$. Let $\mathcal{B}(G)$ be the space consisting of all functions $\varphi$ in $\mathcal{B}\left(\mathbb{D}^{*}\right)$ which are quadratic differentials for $\mathrm{G}$. We can see that $\mathcal{T}(G)$ is a subset of $\mathcal{B}(G)$. 
In 2004, Cui and Zinsmeister [6] proved the following theorem based on the welldefined property of the Douady-Earle extension operator restricted in $S Q S_{*}(\mathbb{S})$.

Theorem A. The mapping $\Psi:[\mu] \mapsto f^{\mu}$ is a bijection from $\mathcal{T}_{S}$ onto $S Q S_{*}(G)$, while $\beta:[\mu] \mapsto \mathcal{S}_{f_{\mu}}$ is bijective from $\mathcal{T}_{S}$ onto $\mathcal{T}(G)$.

One of the goals of this paper is to make this theorem precise using methods in [18] by showing both two maps above are actually homeomorphisms (even biholomorphic automorphisms). Before proceeding we end this section by a discussion on Fuchsian groups leading to non-trivial BMO-Teichmüller spaces

2.3 Groups of convergence type. In contrast to the classical Teichmüller spaces, $\mathcal{T}_{S}$ can be trivial, as shown by Astala and Zinsmeister [4]. For completeness, let us recall some related facts.

Let $G$ be a discrete group of Möbius transformation on $\hat{\mathbb{C}}$. We say that $G$ has the Mostow rigidity property if for each homeomorphism $h: \mathbb{S} \rightarrow \mathbb{S}$ with $h \circ G \circ h^{-1}$ a Möbius group, it holds that either $h$ is completely singular or else is a Möbius transformation.

We say that the group $G$ acting on $\hat{\mathbb{C}}$ is of divergence type if

$$
\sum_{\gamma \in G}(1-|\gamma(0)|)=\infty
$$

and of convergence type, if the series converge. It was shown by Agard [1] and Sullivan [17] that groups of divergence type have the Mostow rigidity property. In 1990, Astala and Zinsmeister [4] proved the converse:

Theorem B. A Fuchsian group $G$ has Mostow rigidity property if and only if it is of divergence type.

Combining with Theorem A, we see that $\mathcal{T}_{S}$ is trivial if $G$ is of divergence type, while $\mathcal{T}_{S}$ is never trivial if $G$ is of convergence type. In what follows, we henceforth shall not deal with Fuchsian groups of divergence type. We shall focus on Fuchsian groups of convergence type.

\section{Complex structure on $\mathcal{T}_{S}$}

In this section, we adopt methods from [18] to prove that $\mathcal{T}_{S}$ has a natural complex Banach manifold structure. As a byproduct, we shall strengthen the above conclusion (Theorem A) by Cui and Zinsmeister.

We begin with some basic definitions and notations. Let $C$ denote the universal constant that might change from one line to another. While $C(\cdot), C_{1}(\cdot), C_{2}(\cdot), \cdots$ will denote constants that depend only on the elements put in the brackets. Denote by $\rho_{D}(z)$ the hyperbolic metric in the Jordan domain $D$. The notation $A \approx B$ means that there exists a universal constant $C$ such that $\frac{B}{C} \leqslant A \leqslant C B$. 
Let $\Omega=f_{\mu}(\mathbb{D})$ and $\Omega^{*}=f_{\mu}\left(\mathbb{D}^{*}\right)$, then $\Omega$ and $\Omega^{*}$ are complementary Jordan regions bounded by a quasicircle. Let

$$
z^{*}=\gamma(\Omega)(z)=f_{\mu} \circ j \circ f_{\mu}^{-1}(z)
$$

be a quasiconformal reflection that fixes $\partial \Omega$ pointwise and interchanges $\Omega$ and $\Omega^{*}$, where $j(z)=\frac{1}{z}$. If $g$ is a Möbius transformation of the extended complex plane, then, according to (2),

$$
\gamma(g(\Omega))=\left(g \circ f_{\mu}\right) \circ j \circ\left(g \circ f_{\mu}\right)^{-1}=g \circ \gamma(\Omega) \circ g^{-1} .
$$

Similar to the Ahlfors map (see [10]), the mapping

$$
\varphi \mapsto \mu_{\Omega}(\varphi)
$$

is defined on the Banach space

$$
B\left(\Omega^{*}\right)=\left\{\varphi \text { is holomorphic in } \Omega^{*}: \sup _{z \in \Omega^{*}}|\varphi(z)| \rho_{\Omega^{*}}^{-2}(z)<\infty\right\}
$$

by putting

$$
\mu_{\Omega}(\varphi)(z)=\frac{\varphi\left(z^{*}\right)\left(z^{*}-z\right)^{2} \gamma(\Omega)_{\bar{z}}(z)}{2+\varphi\left(z^{*}\right)\left(z^{*}-z\right)^{2} \gamma(\Omega)_{z}(z)}, z \in \Omega .
$$

Lemma 1. $\mu_{g(\Omega)}(\varphi)(g(z)) \frac{\overline{g^{\prime}(z)}}{g^{\prime}(z)}=\mu_{\Omega}\left(\varphi \circ g\left(g^{\prime}\right)^{2}\right)(z)$.

Proof. We borrow the method from Earle-Nag [10]. According to (3),

$$
\mu_{g(\Omega)}(\varphi)(g(z)) \frac{\overline{g^{\prime}(z)}}{g^{\prime}(z)}=\frac{\varphi\left(g(z)^{*}\right)\left(g(z)^{*}-g(z)\right)^{2} \gamma(g(\Omega))_{\bar{z}}(g(z))}{2+\varphi\left(g(z)^{*}\right)\left(g(z)^{*}-g(z)\right)^{2} \gamma(g(\Omega))_{z}(g(z))} \frac{\overline{g^{\prime}(z)}}{g^{\prime}(z)} .
$$

Here $g(z)^{*}=\gamma(g(\Omega))(g(z))=g \circ \gamma(\Omega) \circ g^{-1}(g(z))=g \circ \gamma(\Omega)(z)=g\left(z^{*}\right)$, if $z^{*}=\gamma(\Omega)(z)$. Differentiating both sides of

$$
\gamma(g(\Omega))(g(z))=g \circ \gamma(\Omega)(z)
$$

with respect to $\bar{z}$ and $z$ respectively, we find that

$$
\gamma(g(\Omega))_{\bar{z}}(g(z)) \overline{g^{\prime}(z)}=g^{\prime}\left(z^{*}\right) \gamma(\Omega)_{\bar{z}}(z)
$$

and

$$
\gamma(g(\Omega))_{z}(g(z)) g^{\prime}(z)=g^{\prime}\left(z^{*}\right) \gamma(\Omega)_{z}(z) .
$$

Therefore,

$$
\begin{aligned}
\mu_{g(\Omega)}(\varphi)(g(z)) \frac{\overline{g^{\prime}(z)}}{g^{\prime}(z)} & =\frac{\varphi\left(g\left(z^{*}\right)\right)\left(z^{*}-z\right)^{2} g^{\prime}\left(z^{*}\right)^{2} \gamma(\Omega)_{\bar{z}}(z)}{2+\varphi\left(g\left(z^{*}\right)\right)\left(z^{*}-z\right)^{2} g^{\prime}\left(z^{*}\right)^{2} \gamma(\Omega)_{z}(z)} \\
& =\mu_{\Omega}\left(\varphi \circ g\left(g^{\prime}\right)^{2}\right)(z) .
\end{aligned}
$$


Lemma 2 (see [18]). Let $\alpha>0, \beta>0$. For a positive function $\lambda$ in $\mathbb{D}$, set

$$
\tilde{\lambda}(z)=\iint_{\mathbb{D}} \frac{\left(1-|z|^{2}\right)^{\alpha}\left(1-|w|^{2}\right)^{\beta}}{|1-\bar{z} w|^{\alpha+\beta+2}} \lambda(w) d u d v .
$$

Then, using the same notation for a function $\tau$ and the associated measure $\tau d x d y$, we have that $\tilde{\lambda} \in C M(\mathbb{D})$ if $\lambda \in C M(\mathbb{D})$, and $\|\tilde{\lambda}\|_{c} \leqslant C\|\lambda\|_{c}$, while $\tilde{\lambda} \in C M_{0}(\mathbb{D})$ if $\lambda \in C M_{0}(\mathbb{D})$.

Lemma 3 (see [21]). Let $f$ be conformal in $\mathbb{D}$. Then $\log f^{\prime} \in B M O A(\mathbb{D})$ if and only if for each Carleson measure $\lambda \in C M(\Omega), \Omega=f(\mathbb{D})$, the inverse image $\lambda \circ f\left|f^{\prime}\right| \in C M(\mathbb{D})$. Besides, the norm of $\lambda \circ f\left|f^{\prime}\right|$ is dominated by the norm of $\lambda$.

Let us denote by $T(1), \mathcal{T}(1)$, the spaces $T(G), \mathcal{T}(G)$ for $G=\{I\}$, and also denote by $B\left(\mathbb{D}^{*}\right), \mathcal{B}\left(\mathbb{D}^{*}\right)$, the spaces $B(G), \mathcal{B}(G)$ for $G=\{I\}$. We will also need the spaces $B(\mathbb{D})$ and $\mathcal{B}(\mathbb{D})$. $B(\mathbb{D})$ and $\mathcal{B}(\mathbb{D})$ can be defined respectively just $\rho_{\mathbb{D}^{*}}(z)$ replaced by $\rho_{\mathbb{D}}(z)$ in the definitions of $B\left(\mathbb{D}^{*}\right)$ and $\mathcal{B}\left(\mathbb{D}^{*}\right)$. The following Lemma shows that $\mathcal{B}(\mathbb{D}) \subset B(\mathbb{D})$.

Lemma 4. For any $\phi \in \mathcal{B}(\mathbb{D}),\|\phi\|_{B} \leqslant \frac{32 \sqrt{2}}{3}\|\phi\|_{\mathcal{B}}$.

Proof. Examine the proof of Lemma 4.1 in [18] carefully, we can find that this conclusion is valid.

Lemma 5. The space $\mathcal{B}(G)$ is a Banach space, and the set $\mathcal{T}(G)$ is open in $\mathcal{B}(G)$. Furthermore, there exists a constant $k$, so that the ball $B(0, k)=\left\{\varphi \in \mathcal{B}(G):\|\varphi\|_{\mathcal{B}}<\right.$ $k$ \} lies in $\mathcal{T}(G)$.

Proof. We consider functions $\varphi_{n} \in \mathcal{B}(G)$ which converge to $\varphi$ in $\mathcal{B}\left(\mathbb{D}^{*}\right)$. Given a $g \in G$, we then have $\varphi_{n}(z) \rightarrow \varphi(z), \varphi_{n}(g(z)) \rightarrow \varphi(g(z))$, uniformly on every compact subset of $\mathbb{D}^{*}$. It follows that

$$
\varphi(g(z)) g^{\prime}(z)^{2}=\lim _{n \rightarrow \infty} \varphi_{n}(g(z)) g^{\prime}(z)^{2}=\lim _{n \rightarrow \infty} \varphi_{n}(z)=\varphi(z) .
$$

Consequently, $\varphi \in \mathcal{B}(G)$. Therefore, $\mathcal{B}(G)$ is a closed subspace of $\mathcal{B}\left(\mathbb{D}^{*}\right)$ and hence a Banach space.

The definition of $\mathcal{T}(G)$ and Lemma 4 imply that

$$
\mathcal{T}(1)=T(1) \cap \mathcal{B}\left(\mathbb{D}^{*}\right) \subset B\left(\mathbb{D}^{*}\right) \cap \mathcal{B}\left(\mathbb{D}^{*}\right)=\mathcal{B}\left(\mathbb{D}^{*}\right),
$$

so $\mathcal{T}(1)$ is an open subset of $\mathcal{B}\left(\mathbb{D}^{*}\right)$ by the openness of $T(1)$ in $B\left(\mathbb{D}^{*}\right)$. The definition of $\mathcal{T}(G)$ also implies that

$$
\mathcal{T}(G)=\mathcal{T}(1) \cap \mathcal{B}(G) \subset \mathcal{B}\left(\mathbb{D}^{*}\right) \cap \mathcal{B}(G)=\mathcal{B}(G),
$$

so $\mathcal{T}(G)$ is an open subset of Banach space $\mathcal{B}(G)$ by the openness of $\mathcal{T}(1)$ in $\mathcal{B}\left(\mathbb{D}^{*}\right)$.

Now for $\varphi \in \mathcal{B}\left(\mathbb{D}^{*}\right)$, define $\phi=\varphi \circ g\left(g^{\prime}\right)^{2}$, where $z=g(w)=\frac{1}{w}, w \in \mathbb{D}$. Then $\|\phi\|_{B}=\|\varphi\|_{B}$. Let $\lambda_{\phi}(w)=|\phi(w)|^{2}\left(1-|w|^{2}\right)^{3}$ and $\lambda_{\varphi}(z)=|\varphi(z)|^{2}\left(|z|^{2}-1\right)^{3}$ as above. 
then $\lambda_{\phi}\left(\frac{1}{z}\right)\left|\frac{1}{z}\right|^{2}=\lambda_{\varphi}(z), z \in \mathbb{D}^{*}$. So $\|\phi\|_{\mathcal{B}} \approx\|\varphi\|_{\mathcal{B}}$ by Lemma 3. According to Lemma 4, there is a constant $k$, such that $\|\varphi\|_{B} \leqslant \frac{2}{k}\|\varphi\|_{\mathcal{B}}$. Suppose $\|\varphi\|_{B} \leqslant \frac{2}{k}\|\varphi\|_{\mathcal{B}}<2$. Set $\mathcal{S}_{f}=\varphi$. According to Ahlfors-Weill Theorem, $f$ is univalent and can be extended to a quasiconformal mapping of the complex plane with complex dilatation

$$
\mu(z)=-\frac{1}{z} \varphi\left(\frac{1}{\bar{z}}\right)\left(1-|z|^{2}\right)^{2} \frac{1}{(\bar{z})^{4}}, z \in \mathbb{D} .
$$

Lemma 3 implies that

$$
\begin{aligned}
\lambda_{\varphi}\left(\frac{1}{z}\right)\left|\frac{1}{z}\right|^{2} & =\left|\varphi\left(\frac{1}{z}\right)\right|^{2}\left(\frac{1}{|z|^{2}}-1\right)^{3} \frac{1}{|z|^{2}} \\
& =\left|\varphi\left(\frac{1}{z}\right)\right|^{2}\left(1-|z|^{2}\right)^{3} \frac{1}{|z|^{8}}
\end{aligned}
$$

is a Carleson measure in $\mathbb{D}$. Hence

$$
\frac{|\mu(z)|^{2}}{1-|z|^{2}}=\frac{1}{4}\left|\varphi\left(\frac{1}{\bar{z}}\right)\right|^{2}\left(1-|z|^{2}\right)^{3} \frac{1}{|z|^{8}}
$$

is a Carleson measure in $\mathbb{D}$. Consequently, the set $\left\{\varphi \in \mathcal{B}\left(\mathbb{D}^{*}\right):\|\varphi\|_{\mathcal{B}}<k\right\}$ lies in $\mathcal{T}(1)$.

For any $\varphi \in B(0, k)$, since $\mu(z)$ above can be written as

$$
\mu(z)=\frac{1}{z} \varphi\left(z^{*}\right)\left(z-z^{*}\right)^{2} \gamma(\mathbb{D})_{\bar{z}}(z),
$$

where $z^{*}=\gamma(\mathbb{D})(z)=\frac{1}{\bar{z}}$. Then $\varphi \circ g\left(g^{\prime}\right)^{2}=\varphi$ implies $\mu \circ g \frac{\bar{g}^{\prime}}{g}=\mu$ by Lemma 1. So $\mu$ is a Beltrami differential for $G$. Now we can come to the conclusion that for any $\varphi \in B(0, k)$, there exists a holomorphic function $f$ in $\mathbb{D}^{*}$ which can be extended to a quasiconformal mapping in the complex plane whose complex dilatation $\mu \in \mathcal{M}(G)$ and $\mathcal{S}_{f}=\varphi$.

Theorem 1. The function

$$
S: \mu \mapsto \mathcal{S}_{\left.f_{\mu}\right|_{\Delta *}}
$$

which maps $\mathcal{M}(G)$ into $\mathcal{B}(G)$ is holomorphic, and it has local holomorphic sections everywhere in $\mathcal{T}(G)$.

Proof. We first show that $S: \mathcal{M}(G) \rightarrow \mathcal{B}(G)$ is continuous. We borrow some discussion from Astala and Zinsmeister [3]. By an integral representation of the Schwarzian derivative by means of the representation theorem of quasiconformal mappings, Astala and Zinsmeister [3] proved that for any two elements $\mu$ and $\nu$ in $M(\mathbb{D})$, there exists some constant $C_{1}\left(\|\mu\|_{\infty}\right)$ such that

$$
|S(\nu)(z)-S(\mu)(z)|^{2} \leq \frac{C_{1}\left(\|\mu\|_{\infty}\right)}{\left(|z|^{2}-1\right)^{2}} \iint_{\mathbb{D}} \frac{|\nu(\zeta)-\mu(\zeta)|^{2}+\|\nu-\mu\|_{\infty}^{2}|\mu(\zeta)|^{2}}{|\zeta-z|^{4}} d \xi d \eta .
$$


Then,

$$
\begin{aligned}
|S(\nu)(z)-S(\mu)(z)|^{2} & \left(|z|^{2}-1\right)^{3} \\
& \leq C_{1}\left(\|\mu\|_{\infty}\right)\left(\iint_{\mathbb{D}} \frac{|\nu(\zeta)-\mu(\zeta)|^{2}}{1-|\zeta|^{2}} \frac{\left(1-|\zeta|^{2}\right)\left(|z|^{2}-1\right)}{|\zeta-z|^{4}} d \xi d \eta\right. \\
& \left.+\|\nu-\mu\|_{\infty}^{2} \iint_{\mathbb{D}} \frac{|\mu(\zeta)|^{2}}{1-|\zeta|^{2}} \frac{\left(1-|\zeta|^{2}\right)\left(|z|^{2}-1\right)}{|\zeta-z|^{4}} d \xi d \eta\right) .
\end{aligned}
$$

According to Lemma 2, there exists some constant $C_{2}\left(\|\mu\|_{\infty}\right)$ such that

$$
\begin{aligned}
\|S(\nu)-S(\mu)\|_{\mathcal{B}}^{2} & \leq C_{2}\left(\|\mu\|_{\infty}\right)\left(\left\|\lambda_{\nu-\mu}\right\|_{c}^{2}+\|\nu-\mu\|_{\infty}^{2}\left\|\lambda_{\mu}\right\|_{c}^{2}\right) \\
& \leq C_{2}\left(\|\mu\|_{\infty}\right)\left(1+\|\mu\|_{c}^{2}\right)\|\nu-\mu\|_{c}^{2}
\end{aligned}
$$

Consequently, $S: \mathcal{M}(G) \rightarrow \mathcal{B}(G)$ is continuous.

To prove that $S: \mathcal{M}(G) \rightarrow \mathcal{B}(G)$ is a holomorphic map, we use a general result about the infinite dimensional holomorphy (see [14, 15]). It says that a continuous map $f$ from a domain $U$ in a complex Banach space $X$ into another complex Banach space $Y$ is holomorphic if for each pair $(u, x)$ in $U \times X$ and each element $y^{*}$ from a total subset $Y_{0}^{*}$ of the dual space $Y^{*}, y^{*}(f(u+t x))$ is a holomorphic function in a neighborhood of zero in the complex plane. Here a subset $Y_{0}^{*}$ of $Y^{*}$ is total if $y^{*}(y)=0$ for all $y^{*} \in Y_{0}^{*}$ implies that $y=0$.

We have already seen from Lemma 5 that $\mathcal{B}(G)$ is a Banach space. The ball $\mathcal{M}(G)$ is an open subset of the Banach space $\mathcal{L}(G)$. Now for each $z \in \mathbb{D}^{*}$, define $l_{z}(\phi)=$ $\phi(z), \forall \phi \in \mathcal{B}(G)$. The proof of Lemma 5 says that $|\phi(z)|\left(|z|^{2}-1\right)^{2} \leq C\left\|_{\phi}\right\|_{\mathcal{B}}$, which implies that $\left\|l_{z}\right\| \leq C\left(|z|^{2}-1\right)^{-2}$. Thus, $l_{z} \in \mathcal{B}^{*}(G)$. Set $A=\left\{l_{z}: z \in \mathbb{D}^{*}\right\}$. Clearly, A is a total subset of $\mathcal{B}^{*}(G)$. Now for each $z \in \mathbb{D}^{*}$, each pair $(\mu, \nu) \in \mathcal{M}(G) \times \mathcal{L}(G)$ and small $t$ in the complex plane, by the well known holomorphic dependence of quasiconformal mappings on parameters (see [2, 14, 15]), we conclude that $l_{z}(S(\mu+t \nu))=S(\mu+t \nu)(z)$ is a holomorphic function of $t$. Consequently, $S: \mathcal{M}(G) \rightarrow \mathcal{B}(G)$ is holomorphic.

Finally, we prove $S: \mathcal{M}(G) \rightarrow \mathcal{B}(G)$ has local holomorphic sections everywhere in $\mathcal{T}(G)$. Fix $\phi \in \mathcal{T}(G)$. There exists a univalent function $f$ on $\mathbb{D}^{*}$ such that $\mathcal{S}_{f}=\phi$. Let $F$ be a Riemann mapping from $\mathbb{D}$ onto $\mathbb{C} \backslash \overline{f\left(\mathbb{D}^{*}\right)}$ and $h=F^{-1} \circ f$ the conformal welding. Then we have $h \in S Q S(\mathbb{S})$. Now the proof in [14, P.199] gives that $h \in S Q S(G)$. Let $f_{\mu}=F \circ E(h)$. Then $f_{\mu}$ is a quasiconformal extension of $f$ whose dilatation $\mu$ is in $\mathcal{M}(G)$. Thus $S(\mu)=\phi$. Let $\Omega=f_{\mu}(\mathbb{D})$ and $\Omega^{*}=f_{\mu}\left(\mathbb{D}^{*}\right)$. We have $\rho_{\Omega^{*}}\left(f_{\mu}(z)\right)\left|f_{\mu}^{\prime}(z)\right|=$ $\left(|z|^{2}-1\right)^{-1}$ for $z \in \mathbb{D}^{*}$. Consider $U_{\epsilon}(\phi)=\left\{\psi \in \mathcal{B}(G):\|\psi-\phi\|_{\mathcal{B}}<\epsilon\right\}$ for $\epsilon>0$. Then for each $\psi \in U_{\epsilon}(\phi)$ there exists a unique locally univalent function $f_{\psi}$ in $\mathbb{D}^{*}$ with $f_{\mu}(z)=z+\frac{b_{1}}{z}+\cdots$ as $z \rightarrow \infty$ such that $\mathcal{S}_{f_{\psi}}=\psi$. Set $g_{\psi}=f_{\psi} \circ\left(f_{\mu}\right)^{-1}$. Then $\mathcal{S}_{g_{\psi}}=\left((\psi-\phi) \circ f_{\mu}^{-1}\right)\left(\left(f_{\mu}^{-1}\right)^{\prime}\right)^{2}$ and $\sup _{z \in \Omega^{*}} \rho_{\Omega^{*}}^{-2}(z)\left|\mathcal{S}_{g_{\psi}}(z)\right|=\|\psi-\phi\|_{B}$. Since $\psi, \phi \in \mathcal{B}(G), \psi \circ g g^{\prime 2}=\psi$ and $\phi \circ g g^{\prime 2}=\phi$ for any $g \in G$. Hence,

$$
\mathcal{S}_{g_{\psi}} \circ \widetilde{g}(\widetilde{g})^{\prime 2}=\mathcal{S}_{g_{\psi}}
$$


for any $\widetilde{g} \in G_{\mu}$. Since the inclusion map $i: \mathcal{B}(G) \rightarrow B(G)$ is continuous, $\|\psi-\phi\|_{B}<C \epsilon$ for $\psi \in U_{\epsilon}(\phi)$. The Earle-Nag reflection [12, P.263] associated with the curve $\Gamma=\partial \Omega$ is given by the formula

$$
\gamma(z)= \begin{cases}f \circ j \circ f_{\mu}^{-1}(z)=f \circ j \circ E(h)^{-1} \circ F^{-1}, & z \in \Omega \\ z, & z \in \Gamma \\ \gamma^{-1}(z), & z \in \Omega^{*}\end{cases}
$$

where $j(z)=1 / \bar{z}$, and [12, P.265] says

$$
C_{3}^{-1}\left(\|\mu\|_{\infty}\right) \leqslant|\gamma(z)-z|^{2} \rho_{\Omega^{*}}^{-2}(\gamma(z))|\bar{\partial} \gamma(z)| \leqslant C_{3}\left(\|\mu\|_{\infty}\right) .
$$

Under the condition that $\sup _{z \in \Omega^{*}} \rho_{\Omega^{*}}^{-2}(z)\left|\mathcal{S}_{g_{\psi}}(z)\right|$ is sufficiently small (when $\epsilon$ is sufficiently small), Ahlfors [2], Earle and Nag [10] (see also [12, P.266]) proved that $g_{\psi}$ is univalent and can be extended to a quasiconformal mapping in the whole plane whose complex dilatation $\mu_{\psi}$ has the form

$$
\mu_{\psi}(z)=\frac{\mathcal{S}_{g_{\psi}}(\gamma(z))(\gamma(z)-z)^{2} \bar{\partial} \gamma(z)}{2+\mathcal{S}_{g_{\psi}}(\gamma(z))(\gamma(z)-z)^{2} \partial \gamma(z)}, z \in \Omega .
$$

Then by means of ([6) we have

$$
\left|\mu_{\psi}(z)\right| \leqslant C_{4}\left(\|\mu\|_{\infty}\right)\left|\mathcal{S}_{g_{\psi}}(\gamma(z))\right| \rho_{\Omega^{*}}^{-2}(\gamma(z)), z \in \Omega
$$

Consequently, $f_{\psi}=g_{\psi} \circ f_{\mu}$ is univalent in $\mathbb{D}^{*}$ and has a quasiconformal extension to the whole plane whose complex dilatation $\nu_{\psi}$ is

$$
\nu_{\psi}=\frac{\mu+\left(\mu_{\psi} \circ f_{\mu}\right) \tau}{1+\bar{\mu}\left(\mu_{\psi} \circ f_{\mu}\right) \tau}, \quad \tau=\frac{\overline{\partial f_{\mu}}}{\partial f_{\mu}} .
$$

According to Lemma 1, (5) implies that

$$
\mu_{\psi} \circ \widetilde{g} \frac{\overline{(\widetilde{g})^{\prime}}}{(\widetilde{g})^{\prime}}=\mu_{\psi}
$$

Again we know that $\mu \circ g \frac{\overline{g^{\prime}}}{g^{\prime}}=\mu$. By direct computation, we can get

$$
\nu_{\psi} \circ g \frac{\overline{g^{\prime}}}{g^{\prime}}=\nu_{\psi}
$$

Now, it follows from (8) that

$$
\begin{aligned}
\left|\mu_{\psi}\left(f_{\mu}(z)\right)\right| & \leqslant C_{4}\left(\|\mu\|_{\infty}\right)\left|\mathcal{S}_{g_{\psi}}\left(\gamma\left(f_{\mu}(z)\right)\right)\right| \rho_{\Omega^{*}}^{-2}\left(\gamma\left(f_{\mu}(z)\right)\right) \\
& =C_{4}\left(\|\mu\|_{\infty}\right)\left|\mathcal{S}_{g_{\psi}}\left(f_{\mu}(j(z))\right)\right| \rho_{\Omega^{*}}^{-2}\left(\gamma\left(f_{\mu}(z)\right)\right) \\
& =C_{4}\left(\|\mu\|_{\infty}\right)|\psi(j(z))-\phi(j(z))|\left(1-|j(z)|^{2}\right)^{2},
\end{aligned}
$$


which implies that $\left\|\lambda_{\mu_{\psi} \circ f_{\mu}}\right\|_{c} \leqslant C_{5}\left(\|\mu\|_{\infty}\right)\|\psi-\phi\|_{\mathcal{B}}$. Thus, $\mu_{\psi} \circ f_{\mu} \in \mathcal{M}(\mathbb{D})$, and we conclude by (9) that $\nu_{\psi} \in \mathcal{M}(\mathbb{D})$. Combining with (10), we can get $\nu_{\psi} \in \mathcal{M}(G)$. On the other hand, from (7) and (9) it is easy to see that $\nu_{\psi}$ depends holomorphically on $\psi$. Since $S\left(\nu_{\psi}\right)=\psi$, we conclude that $\nu: U_{\epsilon}(\phi) \rightarrow \mathcal{B}(G)$ is a local holomorphic section to $S: \mathcal{M}(G) \rightarrow \mathcal{B}(G)$. This completes the proof.

Corollary 1. Via the Bers embedding $\beta, \mathcal{T}_{S}$ carries a natural complex structure. Under this complex structure, the Bers embedding

$$
\beta: \mathcal{T}_{S} \rightarrow \mathcal{T}(G)
$$

is biholomorphic.

Proof. First of all, $\beta: \mathcal{T}_{S} \rightarrow \mathcal{T}(G)$ is one-to-one by Theorem A.

Next, $S: \mathcal{M}(G) \rightarrow \mathcal{B}(G)$ is continuous by Theorem 1 and $\Phi: \mu \mapsto[\mu]$ which maps $\mathcal{M}(G)$ onto $\mathcal{T}_{S}$ is a projection. Thus, $\beta: \mathcal{T}_{S} \rightarrow \mathcal{B}(G)$ is continuous by the topological property of the projection mapping. We fix a point $\phi \in \mathcal{T}(G)$, consider $U_{\epsilon}(\phi)=\left\{\psi \in \mathcal{B}(G):\|\psi-\phi\|_{\mathcal{B}}<\epsilon\right\}$ for $\epsilon>0$. According to the proof of Theorem 1, there exists a mapping $\nu: \psi \rightarrow \nu_{\psi}$ which maps $U_{\epsilon}(\phi)$ into $\mathcal{M}(G)$, and it is continuous. The projection $\Phi: \mathcal{M}(G) \rightarrow \mathcal{T}_{S}$ is also continuous. So the composition $\beta^{-1}=\Phi \circ \nu$ : $U_{\epsilon}(\phi) \rightarrow \mathcal{T}_{S}$ is continuous. Thus, $\beta: \mathcal{T}_{S} \rightarrow \mathcal{T}(G)$ is a homeomorphism, i.e., $\mathcal{T}_{S}$ is actually homeomorphic to an open subset in the complex Banach space $\mathcal{B}(G)$.

Hence, by using this result, we define on $\mathcal{T}_{S}$ a complex analytic Banach manifold structure. The Bers embedding $\beta: \mathcal{T}_{S} \rightarrow \mathcal{B}(G)$ is biholomorphic under this complex structure.

Corollary 2. The canonical projection

$$
\Phi: \mathcal{M}(G) \rightarrow \mathcal{T}_{S}
$$

is holomorphic, and it has local holomorphic sections everywhere in $\mathcal{T}_{S}$.

Proof. First of all, we have

$$
\Phi=\beta^{-1} \circ S
$$

Since $\beta^{-1}$ and $S$ are holomorphic, it follows that $\Phi$ is holomorphic.

Next, let us consider the mapping $\Phi \circ \nu \circ \beta$ which is the identity on $\beta^{-1}\left(U_{\epsilon}(\phi)\right)$. According to the proof of Theorem 1, $\nu \circ \beta$ is the desired section.

Corollary 3. $\Psi: \mathcal{T}_{S} \rightarrow S Q S(G)$ is a homeomorphism. Consequently, $S Q S(G)$ possesses a complex structure so that $\Psi: \mathcal{T}_{S} \rightarrow S Q S(G)$ is a biholomorphic isomorphism.

Proof. We know that $\Psi: \mathcal{T}_{S} \rightarrow S Q S(G)$ is one-to-one by Theorem A. Shen and Wei [18] have proved that $\Psi: \mathcal{T}_{S} \rightarrow S Q S(G)$ is a homeomorphism when $G=\{I\}$. So, $\Psi$ : $\mathcal{T}_{S} \rightarrow S Q S(G)$ is a homeomorphism for any Fuchsian group $G$. The homeomorphism $\beta \circ \Psi^{-1}: S Q S(G) \rightarrow \mathcal{T}(G)$ endows the spaces $S Q S(G)$ with the structure of complex Banach manifolds modeled on the Banach space $\mathcal{B}(G)$. Naturally, under this complex structure, $\Psi: \mathcal{T}_{S} \rightarrow S Q S(G)$ is a biholomorphic isomorphism. 


\section{Gâteaux-differentiability of $e$ at the origin}

In this section, we will show that the Douady-Earle extension operator $e: S Q S(\mathbb{S}) \rightarrow$ $\mathcal{M}(\mathbb{D})$ is Gâteaux-differentiable at the origin, and compute its differential.

Let $E(h)$ denote the Douady-Earle extension of a quasisymmetric homeomorphism $h$ on $\mathbb{S}$. The definition of $E(h)$ is very simple: given $z \in \mathbb{D}, E(h)(z)$ is the unique $w \in \mathbb{D}$ such that

$$
F_{h}(z, w)=\frac{1}{2 \pi} \int_{0}^{2 \pi} \frac{h\left(e^{i u}\right)-w}{1-\bar{w} h\left(e^{i u}\right)} \frac{1-|z|^{2}}{\left|z-e^{i u}\right|^{2}} d u=0 .
$$

Coming to BMO-Teichmüller theory, Cui and Zinsmeister [6] have shown that if $h \in$ $S Q S(\mathbb{S})$ then, if $\mu$ denotes the complex dilatation of the Douady-Earle extension $E(h)$, it holds that

$$
\frac{|\mu|^{2}(z)}{1-|z|} d x d y
$$

is a Carleson measure in $\mathbb{D}$, which implies that the Douady-Earle extension operator $h \mapsto \mu$ is a bijection from $S Q S(\mathbb{S})$ onto its image in $\mathcal{M}(\mathbb{D})$. Lemma 2 combined with [16, Proposition 4.2] shows continuity at $h=I d$ of the operator, but global continuity is still unknown.

Suppose $X$ and $Y$ are Banach spaces, $U \subset X$ is open, and $G: X \rightarrow Y$. The differential $d G(u ; b)$ of $G$ at $u \in U$ in the direction $b \in X$ is defined as

$$
d G(u ; b)=\lim _{t \rightarrow 0} \frac{G(u+t b)-G(u)}{t}=\left.\frac{d}{d t} G(u+t b)\right|_{t=0} .
$$

If the limit exists for all $b \in X$, and if $d G(u ; \cdot): X \rightarrow Y$ is linear and continuous, then one says that $G$ is Gâteaux differentiable at the point $u$.

In the following we show that the operator $e: h \mapsto \mu$ from $S Q S(\mathbb{S})$ onto its image in $\mathcal{M}(\mathbb{D})$ is Gâteaux differentiable at the origin. In order to make this statement precise we first write, for $h \in S Q S(\mathbb{S}), h\left(e^{i t}\right)=e^{i \phi(t)}$, where $\phi$ is an increasing homeomorphism of $[0,2 \pi]$ such that $\phi^{\prime}(t)$ is a Muckenhoupt weight. The topology of $S Q S(\mathbb{S})$ is the one inherited from $B M O_{\mathbb{R}}(\mathbb{S})$ the space of real-valued $2 \pi$-periodic BMO functions:

$$
d\left(h_{1}, h_{2}\right)=\left\|\log \phi_{1}^{\prime}-\log \phi_{2}^{\prime}\right\|_{B M O} .
$$

Put $b(t)=\log \phi^{\prime}(t)$. There is no loss of generality to assume that $\int_{0}^{2 \pi} b(t) d t=0$ (since any $\varphi \in B M O$ can be identified with $\varphi+\alpha, \alpha$ constant).

Conversely, suppose now that $b \in B M O_{\mathbb{R}}(\mathbb{S})$ with $\int_{0}^{2 \pi} b(t) d t=0$. Let $t>0$ be small. We define $c(t)$ as being the unique real number such that

$$
\int_{0}^{2 \pi} e^{t b(u)-t c(t)} d u=2 \pi
$$

That is

$$
c(t)=\log \left[\left(\frac{1}{2 \pi} \int_{0}^{2 \pi} e^{t b(u)} d u\right)^{\frac{1}{t}}\right] .
$$


We have $\lim _{t \rightarrow 0} c(t)=\frac{1}{2 \pi} \int_{0}^{2 \pi} b(u) d u=0$. Hence we can write $h_{t}\left(e^{i u}\right)=e^{i \phi_{t}(u)}$, where

$$
\begin{aligned}
\phi_{t}(u) & =\int_{0}^{u} e^{t b(v)-t c(t)} d v=u+\int_{0}^{u}\left[e^{t b(v)-t c(t)}-1\right] d v \\
& =u+t \int_{0}^{u} b(v) d v+o(t) .
\end{aligned}
$$

Set $B(u)=\int_{0}^{u} b(v) d v$. Then

$$
h_{t}\left(e^{i u}\right)=e^{i \phi_{t}(u)}=e^{i u}+t i e^{i u} B(u)+o(t) .
$$

For $h_{t} \in S Q S(\mathbb{S})$, set $F_{h_{t}}(z, w)=0$. That is

$$
F(t, z, w)=\frac{1}{2 \pi} \int_{0}^{2 \pi} \frac{h_{t}\left(e^{i u}\right)-w}{1-\bar{w} h_{t}\left(e^{i u}\right)} \frac{1-|z|^{2}}{\left|z-e^{i u}\right|^{2}} d u=0 .
$$

Write $w=E\left(h_{t}\right)(z)=f_{t}(z)$ and denote by $\mu_{t}=\left(f_{t}\right)_{\bar{z}} /\left(f_{t}\right)_{z}$ the complex dilatation of $f_{t}$.

Theorem 2. The Douady-Earle extension operator $e: h \mapsto \mu$ from $S Q S(\mathbb{S})$ onto its image in $\mathcal{M}(\mathbb{D})$ is Gâteaux differentiable at the origin, and the differentiate at the origin of $e$ in the direction $b \in B M O_{\mathbb{R}}(\mathbb{S})$ is

$$
d e(0 ; b)=-\frac{\left(1-|z|^{2}\right)^{2}}{2 \pi i} \int_{0}^{2 \pi} \frac{3 e^{2 i u} B(u)}{\left(1-\bar{z} e^{i u}\right)^{4}} d u .
$$

Remark: We shall specify now what we mean by Gâteaux differentiability at the origin of the Douady-Earle extension operator.

According to the above argument, the origin $u=0$ of the Banach space $B M O_{\mathbb{R}}(\mathbb{S})$ corresponds to the origin $h=I d$ of the space $S Q S(\mathbb{S})$. Then the corresponding complex dilatation $\mu$ is equal to 0 .

Let $t>0$ be small. For any $b \in B M O_{\mathbb{R}}(\mathbb{S})$, the strongly quasisymmetric homeomorphism $h_{t}$ can be defined as before. The complex dilatation $\mu_{t}$ denotes the image of $h_{t}$ under the Douady-Earle extension operator.

By saying that the operator $e: h \mapsto \mu$ from $S Q S(\mathbb{S})$ onto its image in $\mathcal{M}(\mathbb{D})$ is Gâteaux differentiable at the origin, we mean (by definition) that $\left.\frac{d \mu_{t}}{d t}\right|_{t=0}$ exists, and it is linear and continuous in $b$.

Proof. We compute $\mu_{t}$ using the implicit function theorem and the formula $F\left(t, z, f_{t}(z)\right)=$ 0 . We get (writing $F_{z}$ for $F_{z}(t, z, w)$, etc.) the system

$$
F_{\bar{z}}+F_{\bar{w}}\left(\overline{f_{t}}\right)_{\bar{z}}+F_{w}\left(f_{t}\right)_{\bar{z}}=0, \quad \bar{F}_{\bar{z}}+\bar{F}_{w}\left(f_{t}\right)_{\bar{z}}+\bar{F}_{\bar{w}}\left(\overline{f_{t}}\right)_{\bar{z}}=0
$$

whose solution is

$$
\left(f_{t}\right)_{\bar{z}}=\frac{\bar{F}_{\bar{z}} F_{\bar{w}}-F_{\bar{z}} \bar{F}_{\bar{w}}}{\left|F_{w}\right|^{2}-\left|F_{\bar{w}}\right|^{2}}, \quad\left(f_{t}\right)_{z}=\frac{\bar{F}_{z} F_{\bar{w}}-F_{z} \bar{F}_{\bar{w}}}{\left|F_{w}\right|^{2}-\left|F_{\bar{w}}\right|^{2}}
$$


and finally

$$
\mu_{t}=\frac{\left(f_{t}\right)_{\bar{z}}}{\left(f_{t}\right)_{z}}=\frac{\overline{\left(F_{z}\right)} F_{\bar{w}}-F_{\bar{z}} \overline{\left(F_{w}\right)}}{\overline{\left(F_{\bar{z}}\right)} F_{\bar{w}}-F_{z} \overline{\left(F_{w}\right)}} \triangleq \frac{N}{D} .
$$

Therefore,

$$
\left.\frac{d \mu_{t}}{d t}\right|_{t=0}=\left.\left(\frac{1}{D} \dot{N}-\frac{N}{D^{2}} \dot{D}\right)\right|_{t=0}
$$

Recall that

$$
F(t, z, w)=\frac{1}{2 \pi} \int_{0}^{2 \pi} \frac{h_{t}\left(e^{i u}\right)-w}{1-\bar{w} h_{t}\left(e^{i u}\right)} \frac{1-|z|^{2}}{\left|z-e^{i u}\right|^{2}} d u
$$

Then we have

$$
F_{\bar{z}}(t, z, w)=\frac{1}{2 \pi} \int_{0}^{2 \pi} \frac{h_{t}\left(e^{i u}\right)-w}{1-\bar{w} h_{t}\left(e^{i u}\right)} \frac{e^{-i u}}{\left(\bar{z}-e^{-i u}\right)^{2}} d u
$$

and

$$
F_{z}(t, z, w)=\frac{1}{2 \pi} \int_{0}^{2 \pi} \frac{h_{t}\left(e^{i u}\right)-w}{1-\bar{w} h_{t}\left(e^{i u}\right)} \frac{e^{i u}}{\left(z-e^{i u}\right)^{2}} d u
$$

and

$$
F_{\bar{w}}(t, z, w)=\frac{1}{2 \pi} \int_{0}^{2 \pi} \frac{h_{t}\left(e^{i u}\right)\left(h_{t}\left(e^{i u}\right)-w\right)}{\left(1-\bar{w} h_{t}\left(e^{i u}\right)\right)^{2}} \frac{1-|z|^{2}}{\left|z-e^{i u}\right|^{2}} d u
$$

and

$$
F_{w}(t, z, w)=-\frac{1}{2 \pi} \int_{0}^{2 \pi} \frac{1-|z|^{2}}{\left(1-\bar{w} h_{t}\left(e^{i u}\right)\right)\left|z-e^{i u}\right|^{2}} d u .
$$

Noting that $w=E(h)(z)=z$ if $h(z)=z$, we conclude that

$$
\left.F_{\bar{z}}(t, z, w)\right|_{t=0}=F_{\bar{z}}(0, z, z)=0,\left.\quad F_{z}(t, z, w)\right|_{t=0}=F_{z}(0, z, z)=\frac{1}{1-|z|^{2}}
$$

and

$$
\left.F_{\bar{w}}(t, z, w)\right|_{t=0}=F_{\bar{w}}(0, z, z)=0,\left.\quad F_{w}(t, z, w)\right|_{t=0}=F_{w}(0, z, z)=-\frac{1}{1-|z|^{2}} .
$$

Then

$$
\left.N\right|_{t=0}=\left.\left[\overline{\left(F_{z}\right)} F_{\bar{w}}-F_{\bar{z}} \overline{\left(F_{w}\right)}\right]\right|_{t=0}=0 .
$$

Thus we have

$$
\left.\frac{d \mu_{t}}{d t}\right|_{t=0}=\left.\frac{1}{D} \dot{N}\right|_{t=0}=\left.\left(1-|z|^{2}\right)^{-1}\left(\frac{d}{d t} F_{\bar{w}}(t, z, w)+\frac{d}{d t} F_{\bar{z}}(t, z, w)\right)\right|_{t=0} .
$$

We next compute $\left.\frac{d}{d t} F_{\bar{w}}(t, z, w)\right|_{t=0}$ and $\left.\frac{d}{d t} F_{\bar{z}}(t, z, w)\right|_{t=0}$. Let us start by computing $\left.\frac{\partial f_{t}}{\partial t}(z)\right|_{t=0}$. Differentiating $F\left(t, z, f_{t}(z)\right)=0$ and $\overline{F\left(t, z, f_{t}(z)\right)}=0$ with respect to $t$ using the implicit function theorem we get the system

$$
\begin{aligned}
& F_{t}(0, z, z)+\left.F_{w}(0, z, z) \frac{\partial f_{t}}{\partial t}(z)\right|_{t=0}+F_{\bar{w}}(0, z, z) \overline{\left.\frac{\partial f_{t}}{\partial t}(z)\right|_{t=0}}=0 \\
& \overline{F_{t}(0, z, z)}+\left.\overline{F_{\bar{w}}(0, z, z)} \frac{\partial f_{t}}{\partial t}(z)\right|_{t=0}+\overline{\left.F_{w}(0, z, z) \frac{\partial f_{t}}{\partial t}(z)\right|_{t=0}}=0
\end{aligned}
$$


whose solution is

$$
\left.\frac{\partial f_{t}}{\partial t}(z)\right|_{t=0}=\frac{F_{\bar{w}}(0, z, z) \overline{\left(F_{t}(0, z, z)\right)}-\overline{\left(F_{w}(0, z, z)\right)} F_{t}(0, z, z)}{\left|F_{w}(0, z, z)\right|^{2}-\left|F_{\bar{w}}(0, z, z)\right|^{2}} .
$$

By (12) we have

$$
F_{t}(0, z, z)=\frac{1}{2 \pi} \int_{0}^{2 \pi} \frac{(1-z \bar{z})^{2} i e^{2 i u} B(u)}{\left(e^{i u}-z\right)\left(1-\bar{z} e^{i u}\right)^{3}} d u .
$$

It follows from (18) and (20) that

$$
\left.\frac{\partial f_{t}}{\partial t}(z)\right|_{t=0}=\frac{(1-z \bar{z})^{3}}{2 \pi} \int_{0}^{2 \pi} \frac{i e^{2 i u} B(u)}{\left(e^{i u}-z\right)\left(1-\bar{z} e^{i u}\right)^{3}} d u .
$$

The derivative at $t=0$ of the function $F_{\bar{w}}(t, z, w)$ is

$$
\left.\frac{d}{d t} F_{\bar{w}}(t, z, w)\right|_{t=0}=F_{\bar{w} t}(0, z, z)+\left.F_{\bar{w} w}(0, z, z) \frac{\partial f_{t}}{\partial t}(z)\right|_{t=0}+\left.F_{\bar{w} \bar{w}}(0, z, z) \frac{\overline{\partial f_{t}}}{\partial t}(z)\right|_{t=0} .
$$

By means of (15) we have

$$
F_{\bar{w} t}(0, z, z)=\frac{1-|z|^{2}}{2 \pi} \int_{0}^{2 \pi} \frac{i e^{3 i u} B(u)}{\left(1-\bar{z} e^{i u}\right)^{3}}\left(\frac{1}{e^{i u}-z}+\frac{2 \bar{z}}{1-\bar{z} e^{i u}}+e^{-i u}\right) d u
$$

and

$$
F_{\bar{w} w}(0, z, z)=-\frac{z}{\left(1-|z|^{2}\right)^{2}}, \quad F_{\bar{w} \bar{w}}(0, z, z)=0
$$

Then

$$
\begin{aligned}
\left.\frac{d}{d t} F_{\bar{w}}(t, z, w)\right|_{t=0} & =-\frac{1-|z|^{2}}{2 \pi i} \int_{0}^{2 \pi} \frac{e^{3 i u} B(u)}{\left(1-\bar{z} e^{i u}\right)^{3}}\left(\frac{1}{e^{i u}-z}+\frac{2 \bar{z}}{1-\bar{z} e^{i u}}+e^{-i u}\right) d u \\
& +z\left(1-|z|^{2}\right) \frac{1}{2 \pi i} \int_{0}^{2 \pi} \frac{e^{2 i u} B(u)}{\left(e^{i u}-z\right)\left(1-\bar{z} e^{i u}\right)^{3}} d u .
\end{aligned}
$$

Similarly,

$$
\left.\frac{d}{d t} F_{\bar{z}}(t, z, w)\right|_{t=0}=F_{\bar{z} t}(0, z, z)+\left.F_{\bar{z} w}(0, z, z) \frac{\partial f_{t}}{\partial t}(z)\right|_{t=0}+\left.F_{\bar{z} \bar{w}}(0, z, z) \frac{\overline{\partial f_{t}}}{\partial t}(z)\right|_{t=0} .
$$

By (13) we have

$$
F_{\bar{z} t}(0, z, z)=\frac{1-|z|^{2}}{2 \pi} \int_{0}^{2 \pi} \frac{i e^{2 i u} B(u)}{\left(1-\bar{z} e^{i u}\right)^{4}} d u
$$

and

$$
F_{\bar{z} w}(0, z, z)=0, \quad F_{\bar{z} \bar{w}}(0, z, z)=0 .
$$

Therefore,

$$
\left.\frac{d}{d t} F_{\bar{z}}(t, z, w)\right|_{t=0}=-\frac{1-|z|^{2}}{2 \pi i} \int_{0}^{2 \pi} \frac{e^{2 i u} B(u)}{\left(1-\bar{z} e^{i u}\right)^{4}} d u
$$


It follows from (19) (22) and (23) that

$$
\left.\frac{d \mu_{t}}{d t}\right|_{t=0}=-\frac{\left(1-|z|^{2}\right)^{2}}{2 \pi i} \int_{0}^{2 \pi} \frac{3 e^{2 i u} B(u)}{\left(1-\bar{z} e^{i u}\right)^{4}} d u .
$$

It is easy to see $\left.\frac{d \mu_{t}}{d t}\right|_{t=0}$ is linear in $b$.

Finally we need to show continuity, i.e. that

$$
\frac{\left.\left|\frac{d \mu_{t}}{d t}\right|_{t=0}\right|^{2}}{1-|z|^{2}} d x d y \in C M(\mathbb{D})
$$

with norm controlled by $\|b\|_{B M O}^{2}$. Since each $b \in B M O_{\mathbb{R}}(\mathbb{S})$ with $\int_{0}^{2 \pi} b(x) d x=0$ is a real-valued periodic function with period $2 \pi$, the Fourier series of the function $b(x)$ is given by

$$
b(x)=\sum_{n>0} b_{n} e^{i n x}+\sum_{n>0} b_{-n} e^{-i n x}
$$

where

$$
b_{n}=\frac{1}{2 \pi} \int_{0}^{2 \pi} b(x) e^{-i n x} d x, \quad b_{-n}=\frac{1}{2 \pi} \int_{0}^{2 \pi} b(x) e^{i n x} d x .
$$

Since $b$ is real-valued, we have $b_{-n}=\overline{b_{n}}$. Then

$$
b(x)=\sum_{n>0} b_{n} e^{i n x}+\overline{\sum_{n>0} b_{n} e^{i n x}} .
$$

It follows from the relation $B(u)=\int_{0}^{u} b(x) d x$ that $B(u) \in B M O_{\mathbb{R}}(\mathbb{S})$ and

$$
B(u)=-i \sum_{n>0} \frac{1}{n} b_{n} e^{i n u}+\overline{\left(-i \sum_{n>0} \frac{1}{n} b_{n} e^{i n u}\right)} .
$$

Set $F\left(e^{i u}\right)=-i \sum_{n>0} \frac{1}{n} b_{n} e^{i n u}$. Then $B(u)=F\left(e^{i u}\right)+\overline{F\left(e^{i u}\right)}$.

To prove (25), write

$$
\begin{aligned}
\int_{0}^{2 \pi} \frac{e^{2 i u} B(u)}{\left(1-\bar{z} e^{i u}\right)^{4}} d u & =\int_{0}^{2 \pi} \frac{e^{2 i u} F\left(e^{i u}\right)}{\left(1-\bar{z} e^{i u}\right)^{4}} d u+\int_{0}^{2 \pi} \frac{e^{2 i u} \overline{F\left(e^{i u}\right)}}{\left(1-\bar{z} e^{i u}\right)^{4}} d u \\
& =I_{1}+I_{2} .
\end{aligned}
$$

The first term on the right hand side $I_{1}$ equals to 0 by Cauchy Theorem. By Cauchy integral formula we have

$$
I_{2}=\overline{\int_{0}^{2 \pi} \frac{e^{-2 i u} F\left(e^{i u}\right)}{\left(1-z e^{-i u}\right)^{4}} d u}=\overline{-i \int_{\mathbb{S}} \frac{\zeta F(\zeta)}{(\zeta-z)^{4}} d \zeta}=\overline{\frac{\pi}{3}\left((z F(z))^{\prime}\right)^{\prime \prime}} .
$$

Noting that

$$
(z F(z))^{\prime}=F(z)+z F^{\prime}(z)=\left(-i \sum_{n>0} \frac{1}{n} b_{n} z^{n}\right)+\left(-i \sum_{n>0} b_{n} z^{n}\right) \in B M O A(\mathbb{D}) .
$$

By a result of Fefferman and Stein [9], since $z \mapsto z F^{\prime}(z)+F(z)$ is in $B M O A(\mathbb{D})$,

$$
\frac{\left.\left|\frac{d \mu_{t}}{d t}\right|_{t=0}\right|^{2}}{1-|z|^{2}} d x d y=\frac{1}{4}\left|\left((z F(z))^{\prime}\right)^{\prime \prime}\right|^{2}\left(1-|z|^{2}\right)^{3} d x d y \in C M(\mathbb{D})
$$

with norm controlled by $\|b\|_{B M O}^{2}$. 


\section{References}

[1] S. Agard, A geometric proof of Mostow' s rigidity theorem for groups of divergence type, Acta Math., 151(1983), 231-252.

[2] L. Ahlfors, Lecture on Quasiconformal Mappings, Princeton: Van Nostrand, 1966.

[3] K. Astala and M. Zinsmeister, Teichmüller spaces and BMOA, Math. Ann., 289(1991), 613-625.

[4] K. Astala and M. Zinsmeister, Mostow rigidity and Fuchsian groups, C. R. Acad. Sci. Paris Ser. I Math., 311(1990), 301-306.

[5] A. Beurling and L. V. Ahlfors, The boundary correspondence under quasiconformal mappings, Acta Math., 96(1956), 125-142.

[6] G. Cui and M. Zinsmeister, BMO-Teichmüller spaces, Illinois J. of Math., 48(2004), 1223-1233.

[7] A. Douady and C. J. Earle, Conformal natural extension of homeomorphisms of the circle, Acta Math., 157(1986), 23-48.

[8] Y. Fan, Y. Hu and Y. Shen, On strongly quasisymmetric homeomorphisms, Ann. Acad. Sci. Fenn. Math., 42(2017), 921-930.

[9] R. Fefferman and E. Stein, $H^{p}$ spaces of several variables, Acta Math., 129(1972), 137-193.

[10] C. J. Earle and S. Nag, Conformally natural reflections in Jordan curves with applications to Teichmüller spaces, In: Holomorphic Functions and Moduli , In: MSRI Publications, vol. 11, Springer-Verlag, 1988, 179-194.

[11] J. B. Garnett, Bounded Analytic Functions, Academic Press, New York, 1981.

[12] F. P. Gardiner and N. Lakic, Quasiconformal Teichmüller Theory, Mathematical surveys and monographs, vol. 76, Amer. Math. Soc., 2000.

[13] P. W. Jones, Homeomorphisms of the line which preserve BMO, Ark. Mat., 21(1983), 229-231.

[14] O. Lehto, Univalent Functions and Teichmüller Spaces, Springer-Verlag, New York, 1986.

[15] S. Nag, The Complex Analytic Theory of Teichmüller Spaces, Wiley-Interscience, New York, 1988. 
[16] S. Semmes, Quasiconformal mappings and chord-arc curves, Tran. Amer. Math. Soc., 306(1988), 233-263.

[17] D. Sullivan, On the ergodic theory at infinity of an arbitrary discrete group of hyperbolic motions, in: Riemann surfaces and related topics, Ann. of Math., Studies 97, Princeton.

[18] Y. Shen and H. Wei, Universal Teichmüller space and BMO, Adv. Math., 234(2013), 129-148.

[19] P. Tukia, On infinite dimensional Teichmüller spaces, Ann. Acad. Sci. Fenn Ser. AI Math., 3(1977), 343-372.

[20] P. Tukia, Quasiconformal extension of quasisymmetric mappings compatible with a Möbius group, Acta Math., 154(1985), 153-193.

[21] M. Zinsmeister, Les domaines de Carleson (French) [Carleson domains], Mich. Math. J., 36(1989), 213-220. 